\title{
La formación en Derechos Humanos como parte del proyecto ético político del Trabajo Social
}

\author{
The education in human rights as part of the \\ ethical and political project of Social Work
}

Cory Duarte Hidalgo*

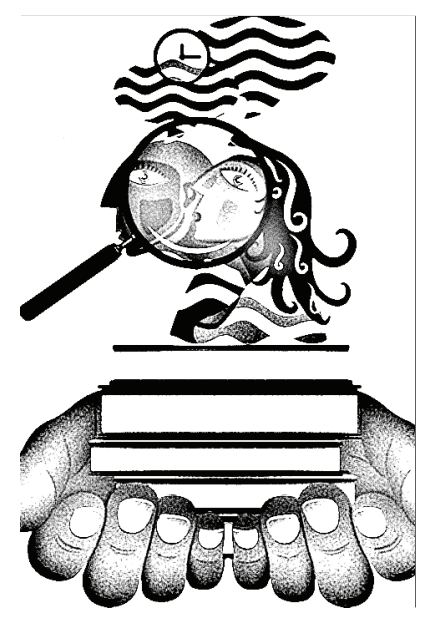

Resumen: La consideración de la formación en Derechos Humanos en tanto elemento primordial del proyecto ético político del trabajo social es el elemento basal de este artículo, el que indaga en los proyectos curriculares de Trabajo Social en Universidades Estatales Chilenas. El estudio de carácter cualitativo, descriptivo y exploratorio, analiza el contenido de estos proyectos permitiéndonos así elaborar recomendaciones para la inclusión de los Derechos Humanos en la política pública de educación superior en general, y en las carreras de trabajo social en particular.

Palabras claves: Formación en Trabajo Social. Derechos Humanos. Educación universitaria.

\begin{abstract}
The education in human rights as a fundamental element of the ethical and political project of Social Work is the base of this article, related to the curriculum projects of Social Work in state Chilean universities. This qualitative, descriptive and exploratory study analyses the content of such projects, so as to lead to recommendations to include human rights in the public policy for college education in general, as well as in the careers in Social Work
\end{abstract}

Keywords: Background in Social Work. Human rights. College education.

* Asistente social, licenciada en Trabajo Social, máster en Trabajo Social Comunitario, máster en Estudios Feministas, máster en Inmigración, refugio y relaciones intercomunitarias, doctoranda en Trabajo Social, Universidad de Atacama, Copiapó, Chile.Email: cory.duarte@uda.cl. 


\section{Presentación}

1 presente artículo revisa la consideración de la formación en derechos
humanos en las carreras de trabajo social de instituciones de educa-
ción superior pertenecientes al Consorcio de Universidades Estata-
les Chilenas (CUECH) ubicadas en la Macrozona Norte del país. Así, se planteó como objetivo de investigación el identificar la presencia o ausencia de la formación en derechos humanos en los perfiles de egreso y mallas curriculares, reflexionando sobre la consideración de la formación en derechos humanos como elemento primordial del proyecto ético político del trabajo social.

Como marco referencial se presenta una somera revisión respecto de la educación en derechos humanos, basándose en los instrumentos internacionales que versan sobre la temática, para luego dar paso a la exploración de los antecedentes que hablan de la inclusión de la formación de los derechos humanos en trabajo social.

Respecto de la forma en que se desarrolla este estudio, se declara su carácter cualitativo, descriptivo y exploratorio, en el cual mediante el análisis de contenido se logran resultados respecto de los objetivos planteados con anterioridad. El documento finaliza con una serie de recomendaciones para la inclusión de la formación de derechos humanos en la política pública de educación superior en general, y en las carreras de trabajo social en particular.

En consideración a lo que más adelante se plantea, la educación en derechos humanos, se transforma en un imperativo de primer orden para las escuelas de trabajo social.

\section{El contexto del estudio: la educación en Derechos Humanos}

La educación por la paz y por los Derechos Humanos ha sido construida con base a múltiples esfuerzos internacionales, los que han sido plasmados en diversos instrumentos, conferencias, encuentros y reuniones. Si bien es cierto, la gran mayoría de las declaraciones y convenciones dedican algunos párrafos a 
la educación en los ámbitos específicos de cada instrumento, existen pactos y declaraciones en las que se plasman en detalle las obligaciones de los Estados al respecto. En este sentido, destaca la Declaración y Programa de acción de Viena (Naciones Unidas, 1993), la cual considera que los Estados partes debiesen incluir "los derechos humanos, el derecho humanitario, la democracia [...] como temas de programas de estudio de todas las instituciones de enseñanza académica y no académica" (art. N. 79). Establece además que la educación en materia de derechos humanos "debe abarcar la paz, la democracia, el desarrollo y la justicia social" (art. N. 80), estipulando que los Estados deben elaborar "programas y estrategias específicos para ampliar al máximo el nivel de educación y difusión de información pública en materia de derechos humanos, teniendo particularmente en cuenta los derechos humanos de la mujer" (art. N. 81).

La Declaración sobre el derecho y el deber de los individuos, los grupos y las instituciones de promover y proteger los Derechos Humanos y las libertades fundamentales universalmente reconocidos, aprobada por la Asamblea General en su Resolución 53/144, de 9 de diciembre de 1998, establece que

Incumbe al Estado la responsabilidad de promover y facilitar la enseñanza de los derechos humanos y las libertades fundamentales en todos los niveles de la educación, y de garantizar que los que tienen a su cargo la formación de abogados, funcionarios encargados del cumplimiento de la ley, personal de las fuerzas armadas y funcionarios públicos incluyan en sus programas de formación elementos apropiados de la enseñanza en derechos humanos. (art. N. 15)

A su vez, en el Decenio de las Naciones Unidas para la educación en la esfera de los derechos humanos, según consta en la Resolución 49/184 del 23 de diciembre de 1994, de la Asamblea General de Naciones Unidas, se fija como objetivo el fomento de la educación en derechos humanos en todos los niveles de enseñanza, así como el establecimiento y fortalecimiento de programas y capacidades para la educación en derechos humanos, entre otros aspectos.

En el caso latinoamericano, se ha exhortado y orientado para que los Estados elaboren planes de acción y diseñen estrategias de educación en derechos humanos. Ejemplo de esto es la conferencia Regional sobre Educación en 
Derechos Humanos (DDHH) en América Latina y el Caribe, realizada el 2001 en Ciudad de México, en la que se define un programa de acción sobre DDHH. En este documento los Estados comprometen la introducción temas de Derechos Humanos, paz y democracia en los planes educativos, incluyendo políticas multiculturales, interdisciplinares y multisectoriales sobre Derechos Humanos, así como la articulación de políticas de igualdad, acciones que deberían ser monitoreadas en forma permanente.

La declaración estipula entre otras cosas garantizar la libertad de cátedra, la instalación de defensorías y la docencia en derechos humanos. Busca que las Universidades desarrollen los derechos humanos en todas las carreras, a través de metodologías sólidas y de carácter plural, fomenten la educación de posgrado en derechos humanos., y que incentiven investigaciones "sobre la realidad nacional y/o regional desde la perspectiva de la población-víctima de violaciones a sus derechos, para conocer las causas que las originan y contribuir en propuestas para su erradicación" (Declaración de México, 2001). Así también, la declaración indica que los Estados deben entregar "la garantía de una formación profesional comprometida con los valores y principios de la democracia para que ello forme parte de los perfiles profesionales y las competencias requeridas en el ejercicio de los mismos" (Declaración de México, 2001).

Resulta interesante la visión de esta declaración en torno a centrarse en el sujeto, reconociendo que "la educación en derechos humanos debe ser un proceso de enseñanza aprendizaje que transforme la vida de las personas e integre lo individual con lo comunitario, lo intelectual con lo afectivo", pero además ha de considerar "el reconocimiento y valoración de la pluralidad cultural presente en la región" (Ramírez, 2004, p. 33).

Los informes especializados identifican 13 instrumentos internacionales que tienen disposiciones atingentes respecto de la consideración de los Derechos Humanos en la Educación (Azúa, 2011, p. 33) en lo que ha implicado que, a partir de la década de los noventa, el Estado Chileno adquiriese compromisos concretos respecto de la Educación en derechos humanos en los distintos niveles educativos.

En el año 2012, en su informe anual el Instituto Nacional de Derechos Humanos (INDH) consignaba la necesidad de considerar los acuerdos suscritos 
por el Estado Chileno en los cuales compromete la inclusión de los derechos humanos en la formación educativa en general y con especial énfasis en la formación universitaria. En el mismo informe el INDH manifiesta que las Universidades no han sido capaces de declarar el compromiso ciudadano con los derechos humanos en las misiones y visiones institucionales. Así también, en el estudio realizado por dicho Instituto respecto de la inclusión de los derechos humanos en las mallas curriculares de pedagogía, derecho y periodismo, se evidencia la inclusión marginal de los derechos humanos en la formación profesional (Azúa, 2011).

En 2011, el Dr. Nash, del Centro de Derechos Humanos de la Facultad de Derecho de la Universidad de Chile denunciaba la escasa importancia de los derechos humanos en las misiones y mallas curriculares de las carreras de derecho a nivel nacional. El académico señala las deficiencias en los currículos en torno a materias relacionadas con la teoría general de derechos humanos, los instrumentos internacionales, la jurisprudencia internacional y los grupos en condición de vulnerabilidad (Nash, 2011, p. 63).

Como resultado del estudio señalado se recomienda la inclusión en los currículos de las carreras del área, el estudio de los grupos protegidos a nivel internacional a través de convenciones específicas, es decir, la consideración de los derechos de las mujeres, niños, niñas y adolescentes, migrantes, diversidades sexuales, pueblos originarios, personas privadas de libertad y refugiados (Nash, 2011, p. 63). En el caso de la teoría general de los derechos humanos, el mismo estudio sugiere su abordaje en asignaturas específicas, pero también en cátedras como Derecho Procesal Penal y Derecho Internacional Público.

Por no encontrarse material respecto de la incorporación de los derechos humanos en las mallas curriculares de trabajo social se hace relevante estudiar respecto de esto, teniendo en cuenta que el objetivo central de la educación en derechos es empoderar a las personas para sean sujetos de derecho, avanzando hacia la consolidación de la justicia y la paz en nuestras sociedades (Mujica, 2002). La educación en derechos humanos es una propuesta ético política que implica la consideración de la teoría general de derechos humanos, pero también la atención de las desigualdades y los grupos que las sufren. De esta forma, los perfiles de egreso permiten formar profesionales sujetos de 
derechos, lo que en resumidas cuentas significa entregar las competencias necesarias para que desarrollen su acción profesional siendo capaces de promocionar y defender sus derechos y los de las personas, grupos y comunidades con quienes trabajan.

\section{Algunas consideraciones sobre la formación en Derechos Humanos en Trabajo Social}

En el ámbito internacional, la Federación Internacional de Trabajo Social (FITS) reconoce desde sus inicios la estrecha interrelación existente entre derechos humanos y trabajo social, lo que se ha consolidado al figurar como entidad consultiva en materias relacionados con derechos sociales, infancia, salud ente otros (Naciones Unidas, 1995). En el año 1992, el Centre for Human Rights de las Naciones Unidas, en conjunto con la International Federation of Social Workers (IFSW/FITS) y la International Association of Schools of Social Work (IASSW), publican por primera vez un manual dedicado a las Escuelas de "Servicio Social", en el que se considera imperativo "que las personas que participan en la enseñanza y la práctica del servicio social se comprometan claramente y sin reservas en la promoción y la protección de los derechos humanos y en la satisfacción de las aspiraciones sociales fundamentales" (p. 11). En el mismo documento se insta a las escuelas de trabajo social a introducir los derechos humanos en sus propuestas curriculares:

Algunas escuelas podrán ofrecer un curso facultativo separado, otras exigir a sus alumnos que sigan un curso de derechos humanos y otras introducir el estudio de los derechos humanos en todos sus cursos fundamentales obligatorios. Esos métodos no tienen por qué excluirse mutuamente, ya que cada uno de ellos tiene ventajas y desventajas. (p. 12)

Así también, en dicho manual, se señala la necesaria coherencia en los programas de trabajo social, los que debiesen reflejar "dimensiones de los derechos humanos y la justicia social" (p. 12). Considerando lo anterior, se señala 
la necesidad de contar con misiones y principios en las escuelas que den cuenta de estas dimensiones, así como las instalación de relaciones y procedimientos con base en los derechos humanos.

En la Declaración del 2000, la FITS reconoce a los derechos humanos y la justicia social como principios de la profesión, relevando su importancia en el proyecto ético político del trabajo social. Reconocer lo anterior implica pensar los derechos consignados en la Declaración Universal como mínimos éticos en el desarrollo de los pueblos, constituyéndose en tanto "condiciones de posibilidad de realización y de transformación con vistas a conseguir una mayor justicia y solidaridad" (Cordero, Palacios y Fernández, 2006, p. 7). Sin embargo, la concepción de derechos de la Declaración Universal resulta problemática en atención a los cuestionamientos sobre la visión universalista, burguesa, androcéntrica y eurocentrista contenida en ella; ante lo cual se hace necesario ligar la idea de dignidad humana con el reconocimiento de múltiples contextos, situados, fundados y multiculturales. Por estas razones, la propuesta de la FITS y la AIETS respecto de la definición de Trabajo Social posee un gran número opositores respecto de la visión etnocéntrica, funcionalista y eurocéntrica de la misma, imponiéndose "los parámetros de principios éticos amplios" (AIETS, 2003), lo que de todas formas, deja a un lado los contextos y especificidades propias de cada región. Las distintas asociaciones regionales, han trabajo en sendas propuestas de modificación de la definición de 2000, las que no han logrado acuerdo ni los consensos necesarios.

En el año 2004, la FITS lista siete instrumentos de derechos humanos considerados de especial relevancia para la práctica y acción del trabajo social (FITS / AIETS, 2004, p. 3), entre estos se encuentran la Declaración Universal, el Pacto de Derechos civiles y políticos (PIDCP), el Pacto de Derechos Económicos, Sociales y Culturales (PIDESC), la Convención por la Eliminación de todas las Formas de Discriminación Racial; la Convención por la Eliminación de todas las formas de discriminación contra la Mujer (CEDAW), la convención de los Derechos del Niño (CDN), y la convención sobre pueblo indígenas y tribales (convenio de la OIT, n. 169). En el mismo año la FITS compromete sus esfuerzos para denunciar y contribuir en la eliminación de toda forma de vulneraciones a los derechos humanos. 
En el caso chileno, el Consorcio de Escuelas de Trabajo Social compuesto por algunas Universidades del CUECH en $2006^{1}$ trabajó en una propuesta colectiva de perfil académico, proceso en el cual se revisaron los ámbitos distintivos de desempeño profesional y los núcleos históricos de formación profesional. En dicho trabajo se reconoce la pertinencia de las competencias concernientes a los derechos humanos en los ámbitos relacionados con sujetos y procesos. En la sistematización realizada por Castañeda y Salamé mencionan que los y las profesionales de lo social se enfrentan a desafíos relacionados con transformaciones estructurales relacionadas con la tecnología y la economía, en un sistema social que se ha complejizado, en el que las respuestas a las demandas corren el riesgo de quedar obsoleta, en lo que las autoras identifican como una crisis de sentido. Así, "la sociedad contemporánea demanda nuevas exigencias a los profesionales del ámbito social, cuestionando los contenidos de su formación y los desempeños laborales que le han sido tradicionales" (Castañeda y Salomé, 2009, p. 3).

Esas nuevas exigencia se dan por las características contextuales de cada escuela de trabajo social y su conexión con el medio local en el que estén insertas; así, materias como migraciones, medio ambientes o movimientos sociales cobran una trascendencia especial debido a las particularidades regionales/ locales, lo que de alguna forma u otra inciden en la formación entregada.

Podemos afirmar que los derechos humanos y su incorporación en trabajo social atienden a una visión ético política de la profesión y disciplina, en la que se considera como perspectiva ética la noción de derechos humanos en toda su complejidad. De esta forma, el principal desafío para las escuelas es la forma de implementar esta perspectiva, evitando caer en una concepción mítica, enfrentándoles como una realidad efectiva (Martín, Esteban y Ramos, 2006), de alcance y complejidad situada.

La consideración de los derechos humanos como marco axiológico de intervención, praxis e investigación en trabajo social, implica adoptarlos en su

1. Este trabajo fue realizado en el marco del proyecto MECESUP UCM 0401, en el cual participaron las siguientes Universidades: Universidad Católica del Maule, Universidad de La Frontera, Universidad de Concepción, Universidad del Bío Bío, Universidad de Antofagasta, Universidad Tecnológica Metropolitana, Universidad Católica de Temuco y Universidad de Los Lagos. 
complejidad e historicidad, facilitando, a través de ellos, procesos de subjetivación de las personas y los pueblos, componente esencial del proyecto ético político del trabajo social. Desde este marco, la consideración de los derechos humanos en las acciones transformadoras de los y las trabajadoras sociales implica la realización de investigaciones e intervenciones respetuosas de la diversidad, democráticas, situadas y críticas. Así, los Derechos Humanos constituyen el guión emancipatorio de nuestra acciones, en torno a un "diálogo intercultural sobre la dignidad humana que eventualmente puede conducir a una concepción mestiza de los derechos humanos, una concepción que en lugar de recurrir a falsos universalismos, se organice como una constelación de significados locales" (Santos, 2002, p. 69-70).

\section{4. ¿A qué apela el proyecto ético político del Trabajo Social?}

Puesto que somos ciudadanos y ciudadanas y nos desempeñamos como tales, inmersos en un sistema democrático en el cual el derecho a tener derechos debiera de considerarse la consigna primordial, las profesiones de lo social requerimos de un marco ético para nuestro actuar, una ética aplicada, puesto que la cotidianeidad de los complejos escenarios en los que nos situamos nos hacen enfrentar dilemas frente a los cuales no existen soluciones estandarizadas, requiriendo de mínimos éticos, pero también, el ejercicio ético de nuestras vocaciones (Cortina, 2002). De esta forma la consideración de la ética aplicada en profesiones como la nuestra permite "orientar de forma mediata, ofreciendo un marco reflexivo para la toma concreta de decisiones" (Cortina, 1996, p. 121).

Considerando esta concepción de ética aplicada, creemos relevante aclarar lo que entendemos en este documento por proyecto ético político del trabajo social. Para esto seguiremos la reflexión de Montaño, respecto de la crisis en la "base de sustentación funcional-laboral" de la profesión, crisis que obliga a reaccionar a través de propuesta de acción de orden colectivo, entre las que el autor señala la necesidad de "construcción/consolidación [...] de un proyecto profesional hegemónico que integre la dimensión ética y la dimensión política" (Montaño, 2005, p. 7), este ha de ser construido en forma democrática por el 
colectivo profesional, "inspirado y articulado a proyectos societarios". Como propuesta, Montaño plantea un proyecto profesional progresista fundado en diversos valores y principios, entre los que destacan los derechos humanos (Montaño, 2005, p. 8). Para el desarrollo de dicho proyecto el autor propone una agenda que considera cuatro elementos: la organización profesional; un marco legal profesional en el que se plasme la operatividad del proyecto y la propuesta; en dicho marco han de estar señalados el reglamento de ejercicio profesional y el código de ética (Montaño, 2005, p. 9); la formación profesional, la que procuraría la "unificación de niveles de formación", "planes de estudios básicos", posgrados y la "promoción de las condiciones para la producción bibliográfica teórica de calidad"; y por último, Montaño considera la articulación con las fuerzas vivas de la sociedad.

En este sentido, si comprendemos a los y las profesionales del trabajo social como sujetos y sujetas que desde sus contextos, historicidades y diversidades enfrentan las distintas realidades sociales en las que están inmersos, elementos como la formación profesional darán contenido a su praxis profesional (Olaya, 2009). Así, el proyecto ético político exigido por Montaño y otros de similares características implican una propuesta que "garantiza compromiso y responsabilidad social, permitiendo que el sujeto sea crítico frente a los desafíos que impone la propuesta del relativismo y la neutralidad y lo más importante, posibilita que se vincule a un proyecto de sociedad radicalmente democrático" (Olaya, 2009, p. 7).

\section{Definición del problema de investigación}

Concluida la presentación de los elementos referenciales que sustentan el estudio realizado corresponde asentar aquellos que permiten plantear el problema de investigación. Entendiendo la conformación tradicional de construcción de este apartado se presentarán el objetivo de investigación, las preguntas guías y la justificación necesaria.

El objetivo principal de este estudio es comparar los perfiles de egreso y mallas curriculares de cuatro carreras de trabajo social pertenecientes al $\mathrm{CUECH}$, 
ubicadas en la Macrozona norte, en relación a la consideración de la formación en derechos humanos como elemento primordial del proyecto ético-político de la profesión.

Considerado lo anterior, los objetivos específicos tienen relación con la pregunta de investigación que guía el análisis: ¿cuáles son los énfasis identificables en los perfiles de egreso y mallas de estudios de las carreras de trabajo social pertenecientes al CUECH en la Macrozona norte, respecto de la formación en derechos humanos?

Por tanto, los objetivos específicos fueron:

a) Identificar la presencia o ausencia de la formación en derechos humanos en los perfiles de egreso y mallas curriculares.

b) Reflexionar sobre la consideración de la formación en derechos humanos como parte del proyecto ético del trabajo social.

c) Proponer algunas recomendaciones para la inclusión de la formación de derechos humanos en la política pública de educación superior en general, y en las carreras de trabajo social en particular.

Dado lo anterior, se configuran como supuestos de este estudio el que los distintos énfasis identificados en la formación en Derechos Humanos afectan a las configuraciones y representaciones sociales de los egresados y egresadas de trabajo social de las universidades consideradas, así, los énfasis formativos influirían de alguna forma en la disposición que tengan los y las profesionales a la hora de realizar investigaciones e intervenciones. De esta forma, si la formación en derechos humanos se convierte en el contenido valórico explícito en el cual se comprometen las instituciones, la carencia de este contenido afectaría a la construcción de un proyecto ético profesional, de las características establecidas por Montaño.

La ausencia de la formación en Derechos Humanos afectaría a los y las egresadas, pero también, afectaría directamente a la conformación del proyecto ético político, puesto que perdería las bases fundacionales del mismo.

Esta situación resulta de interés profesional, disciplinar y gremial puesto que se requieren de identificar competencias mínimas en la formación de 
trabajadores y trabajadoras sociales, las que han de estar respaldadas por las discusiones internacionales realizadas en ese orden, pero también por la contextualización y situación de las unidades académicas en función de las necesidades y demandas locales y regionales.

\section{La inclusión de los derechos humanos en los perfiles de egreso y mallas curriculares de las carreras de trabajo social en las universidades del CUECH en la macrozona norte}

Brevemente mencionaremos que, para lograr conocer y comprender acerca del tema, se utilizó como base un enfoque metodológico cualitativo. Se optó por este enfoque, por considerarse una perspectiva que genera conocimiento acerca de la realidad social, incorporando en las formas de conocer múltiples perspectivas eminentemente éticas y políticas (Tójar, 2006, p. 145), con las que se pretende comprender una realidad múltiple, dinámica y holística.

Siguiendo esta línea, la investigación cualitativa construye conocimientos inclusivos, históricos, contextuales, provisionales, reflexivos, multimetódicos, sistemáticos, subjetivos y holísticos, lo cual potencia y enriquece los procesos de generación y búsqueda de conocimientos emprendidos desde las ciencias sociales, con la finalidad de comprender, investigar (para transformar) interpretar y reflexionar, realidades de características diversas y divergentes.

Dicho esto, expondremos brevemente el método de análisis realizado respecto de la inclusión de los derechos humanos en los planes de estudios de la carrera de trabajo social en las Universidades del Consorcio de Universidades Estatales de Chile, en la macrozona norte, zona comprendida entre las ciudades de Arica y La Serena, analizando la información disponible en las web site de las Universidades de Tarapacá, rturo Prat, de Antofagasta y de Atacama.

Para efectos de la reflexión desplegada, se ha tomado como unidad de análisis los perfiles de egreso y mallas de estudio de las casas de estudio antes señalada, los cuáles han sido trabajados a través de la técnica de análisis de contenido. 


\section{Método: el análisis de contenido}

El análisis de contenido como técnica de investigación en ciencias sociales indaga en la naturaleza de los discursos, permitiendo "formular, a partir de ciertos datos, inferencias reproducibles y válidas que puedan aplicarse a un contexto" (Krippendorff, 1990, p. 82). Esta técnica obliga a quien investiga a situarse desde una triple perspectiva, considerando los datos, su contexto y los marcos interpretativos propios del investigador o investigadora (Porta y Silva, 2003, p. 2). Así, según lo planteado por Raigada, llamamos análisis de contenido al "conjunto de procedimientos interpretativos de productos comunicativos" (Raigada, 2002, p. 2).

Resulta interesante ahondar en la presentación que realiza el mismo autor respecto de los significados de este tipo de análisis:

[...] su propia denominación de análisis de "contenido", lleva a suponer que el "contenido" está encerrado, guardado, incluso a veces oculto dentro de un "continente" (el documento físico, el texto registrado, etc.) y que analizando "por dentro" ese continente", se puede desvelar su contenido (su significado, o su sentido), de forma que una nueva "interpretación" tomando en cuenta los datos del análisis, permitiría un diagnóstico, es decir, un nuevo conocimiento (gnoscere “conocer" a través de su penetración intelectual. (Raigada, 2002, p. 2)

De esta forma, el análisis de contenido logra la emergencia del sentido latente que procede de las prácticas sociales (Raigada, 2002, p. 7), por lo que no sólo interesa indagar respecto de los datos visibles en los documentos comunicativos, sino también en aquellos aspectos no dichos en el discurso.

En este estudio se utilizaron unidades temáticas de análisis, las que permitieron conducir la reflexión a partir de los conceptos, temas y referencias utilizadas o enunciadas en los perfiles de egreso y malla analizados. Ahora bien, para efectos de esta investigación sólo se consideraron aquellos conceptos y temas relacionados con el objeto de estudio. De esta forma, se ha elaborado una serie de categorías, las cuales cumplieron con los requerimientos planteados por Berelson (1967, apud López Noguero, 2011, p. 176), en la cual quien 
investiga razona los motivos de categorización observando los criterios de para ellos establecidos.

Este tipo de análisis se complementa con el enfoque de análisis de marcos de política los cuales son entendidos por Mieke Verloo como un "principio organizador que transforma la información fragmentaria o incidental en un problema de estructura y sentido, en la que una solución está implícita o explícitamente incluido" (Verloo, 2005, p. 20). En este sentido, el análisis de marcos de política puede contribuir a la identificación de la coherencia entre un problema y su solución, y cómo ambos elementos están presentes en los textos políticos (Verloo y Lombardo, 2007).

Uno de los aspectos relevantes de este enfoque es la identificación de las ausencias en el discurso político, además, permite identificar prejuicios "que pueden, sin proponérselo formar los discursos políticos y, en consecuencia, se puede revelar inconsistencias latentes, o incluso los prejuicios" (Verloo y Lombardo, 2007). Asimismo, este tipo de análisis permite identificar las exclusiones existentes en la formulación de las políticas, visibilizando la forma en que "las estrategias discursivas pueden modificar el proceso en sí mismo por medio de la exclusión de algunos actores del debate" (Triandafyllidou y Fotiou, 1998, p. 6.4 apud Verloo y Lombardo, 2007, p. 29).

Así, y considerando el carácter exploratorio y descriptivo de este estudio, en los apartados siguientes se expondrán los análisis preliminares.

\section{Análisis}

Desconocemos los procesos que configuraron los planes de estudios y respectivas mallas de las escuelas de trabajo social de la macrozona norte, sin embargo, podemos realizar análisis acudiendo a la información que cada casa de estudio ha considerado estratégica de difundir. De esta forma los análisis primarios tienen relación con la consideración de los Derechos Humanos en los perfiles de egreso tal y como se grafica a continuación. 
Tabla 1. Categorías de contenidos Nivel 1: Consideración Derechos Humanos en los perfiles de egreso.

\begin{tabular}{|c|c|c|c|c|}
\hline \multirow{2}{*}{ Categorías } & \multicolumn{4}{|c|}{ Universidades } \\
\hline & 1 & 2 & 3 & 4 \\
\hline $\begin{array}{l}\text { Consideración } \\
\text { Derechos Humanos } \\
\text { en forma explícita } \\
\text { (enfoque de } \\
\text { derechos) }\end{array}$ & $\begin{array}{l}\text { No de forma } \\
\text { explícita, pero } \\
\text { hace referencia a } \\
\text { la calidad de vida } \\
\text { de las personas, } \\
\text { grupos, } \\
\text { organizaciones y } \\
\text { comunidades. }\end{array}$ & $\begin{array}{l}\text { No. Pero sí hace } \\
\text { referencia al } \\
\text { empoderamiento y } \\
\text { la superación de } \\
\text { situaciones } \\
\text { conflictivas que } \\
\text { afectan a personas, } \\
\text { familias, grupos y } \\
\text { comunidades. }\end{array}$ & $\begin{array}{l}\text { No hace referencia } \\
\text { directa. Sin } \\
\text { embargo, se } \\
\text { pronuncia en el } \\
\text { reconocimiento de } \\
\text { diversidad, habla } \\
\text { de diferentes } \\
\text { sujetos y territorios } \\
\text { elementos }\end{array}$ & $\begin{array}{l}\text { Sí. Declara como } \\
\text { sello distintivo el } \\
\text { enfoque de } \\
\text { derechos }\end{array}$ \\
\hline $\begin{array}{l}\text { Referencia a } \\
\text { derechos civiles y } \\
\text { políticos }\end{array}$ & No & No & No & No \\
\hline $\begin{array}{l}\text { Referencia a } \\
\text { derechos } \\
\text { económicos, } \\
\text { sociales y } \\
\text { culturales }\end{array}$ & No & No & Sí & Sí \\
\hline $\begin{array}{l}\text { Referencias a } \\
\text { personas o } \\
\text { colectivos "sujetos } \\
\text { de derechos" }\end{array}$ & No & No & Sí & $\begin{array}{l}\text { Sí, declara } \\
\text { ponerles en el } \\
\text { centro. }\end{array}$ \\
\hline $\begin{array}{l}\text { Referencias a } \\
\text { vulneraciones de } \\
\text { derecho }\end{array}$ & No & No & No & $\begin{array}{l}\text { Sí. Se refiere a } \\
\text { las desigualdades } \\
\text { como efecto de } \\
\text { procesos socio } \\
\text { históricos }\end{array}$ \\
\hline $\begin{array}{l}\text { Referencias a la } \\
\text { ética }\end{array}$ & No & No & $\begin{array}{l}\text { Sí. Habla respecto } \\
\text { del compromiso } \\
\text { ético y } \\
\text { reconocimiento de } \\
\text { la diversidad }\end{array}$ & $\begin{array}{l}\text { Sí. Se hace } \\
\text { referencia al } \\
\text { compromiso } \\
\text { ético, y al } \\
\text { fomento de la } \\
\text { autonomía y } \\
\text { protagonismo de } \\
\text { las personas }\end{array}$ \\
\hline
\end{tabular}

Elaboración propia con base en los perfiles de egreso de las unidades académicas señaladas. 
Los perfiles de egreso suelen entenderse como una declaración formal que realizan las instituciones de educación superior, a través de sus carreras profesionales, en las cuales asumen el compromiso formativo contraído y constituyen los aspectos característicos de la profesión en dicha institución, identificando los principales ámbitos de realización y las competencias claves. De esta forma, estos perfiles se configuran como declaraciones de carácter político en los que se presentan los compromisos asumidos por las universidades en cuanto a los límites de la habilitación profesional y/o académica entregada a los y las egresadas de las respectivas carreras. En otras palabras, los perfiles de egreso constituyen el compromiso social de la institución de educación superior en el logro de las competencias, pero también en una obligación que ha de ser demandada por la sociedad "en un real desplazamiento de poder hacia lasociedad que ahora puede y debe reclamar el cumplimiento de las promesas hechas-poner en claro la intencionalidad del programa-explicitar (..) los itinerarios formativos" (Hawes, 2012, p. 2)

Así, y con base en lo anterior, podemos señalar que en las declaraciones realizadas por las Universidades en las carreras de trabajo social, no se hace consideración a la formación en derechos humanos, o a la perspectiva en derechos, o cualquier otra noción que pueda significar alguna consideración al tema. Resulta interesante analizar esta ausencia, ya que a pesar de que en términos profesionales se reconoce su importancia en el currículo, esta no es una competencia con las que contarían quienes egresan de estas instituciones.

En consideración del compromiso ético, que podría de alguna forma solventar la deficiencia anterior, se observa su presencia sólo en dos de estos perfiles, lo que resulta paradójico en cuanto a la consideración de las características propias del trabajo social.

Pese a esto, nos encontramos con una dificultad en la realización de este análisis, puesto que entendemos que los perfiles de egreso son documentos más amplios que los mostrados en las web sites, sin embargo, nos interesan estas declaraciones puesto que son las observables por quienes postulan a las casas de estudios. En esa medida, podríamos especular respecto de la estrategia que pueden asumir las instituciones respecto de los elementos que podrían 
ser atractivos para quienes "eligen" cada una de las carreras. Los perfiles de egreso revisados entregan especial énfasis en los campos laborales en los que cada egresado o egresada podría situarse, haciendo un guiño a las necesidades de mercado por sobre las competencias éticas que cada trabajador o trabajadora social pudiese tener. Complementario a lo anterior, es el énfasis puesto en los aspectos metodológicos y técnicos que perfilan la formación, recordándonos los tiempos en que la profesión se caracterizaba por su mirada tecnocrática, aséptica y neutral. De esta forma, cabe preguntarse entre la coherencia entre estos perfiles esbozados y las mallas de estudios definidas, punto que se tratará a continuación.

Tabla 2. Categorías de contenidos Nivel 2: Consideración Derechos Humanos en la malla curricular.

\begin{tabular}{|c|c|c|c|}
\hline \multirow[t]{2}{*}{ Universidades } & \multicolumn{3}{|c|}{ Categorías } \\
\hline & $\begin{array}{l}\text { Presencia } \\
\text { ausencia } \\
\text { Asig. DDHH }\end{array}$ & $\begin{array}{c}\text { Asignaturas relacionadas } \\
\text { con derechos civiles y } \\
\text { políticos }\end{array}$ & $\begin{array}{l}\text { Asignaturas relacionadas } \\
\text { con derechos económicos, } \\
\text { sociales y culturales }\end{array}$ \\
\hline 1 & Ausente & $\begin{array}{l}\text { Introducción al Derecho. } \\
\text { asignatura derecho de } \\
\text { familia y menores }\end{array}$ & $\begin{array}{l}\text { Asignaturas sociedad y población } \\
\text { indígena/ecología y gestión } \\
\text { ambiental/desarrollo local }\end{array}$ \\
\hline 2 & $\begin{array}{l}\text { Presente } \\
\text { DDHH y TS }\end{array}$ & Derecho laboral y familia & TS Etnias e inmigrantes \\
\hline 3 & Ausente & $\begin{array}{l}\text { Derecho de familia/ } \\
\text { Derecho laboral y } \\
\text { seguridad Social }\end{array}$ & $\begin{array}{l}\text { Teoría del desarrollo social y regional/ } \\
\text { Movimientos sociales y ciudadanía/ } \\
\text { Pobreza y estrategias de intervención. }\end{array}$ \\
\hline 4 & $\begin{array}{l}\text { Presente } \\
\text { DDHH y TS }\end{array}$ & $\begin{array}{l}\text { Marco jurídico para la } \\
\text { intervención social }\end{array}$ & $\begin{array}{l}\text { Ciudadanía y movimientos sociales/ } \\
\text { Desarrollo local. } \\
\text { Optativos temáticos migraciones, } \\
\text { género, medio ambiente, etc. }\end{array}$ \\
\hline
\end{tabular}

Elaboración propia con base en los perfiles en las mallas de estudio de las unidades académicas señaladas. 
Entendiendo los perfiles de egreso como la proyección de la política educativa de cada unidad (Escuela) y sus mallas de estudio como su representación gráfica, podemos señalar que en las unidades analizadas es posible encontrar una representación implícita o explícita de un diagnóstico, conectado a un pronóstico y a un llamado a la acción (Verloo, 2005). En este sentido, las unidades académicas proyectan su formación como más cercana a los derechos sociales, económicos y culturales que a los de orden civil o político, estableciendo una especial atención hacia ciertos colectivos como migrantes, pero también a los contextos en los cuáles desarrollan su acción formativa.

En este sentido, las asignaturas relativas a los derechos civiles y políticos, son las que tradicionalmente han estado presentes en las configuraciones curriculares del trabajo social desde el año 1925, manteniendo incluso la nomenclatura relacionadas con "lo laboral" y "lo familiar".

Retomando a Verloo, la inclusión de asignaturas relacionadas con los Derechos Económicos, Sociales y Culturales (DESC) y los Derechos Civiles y Políticos (DCYP) remite a un pronóstico de necesidad de formación en dichos ámbitos. Sin embargo, su presencia es residual en la estructura curricular. Las asignaturas relacionadas con las otras dimensiones del trabajo social absorben la formación explícita en estas materias.

Llama la atención la inclusión de asignaturas relacionadas con los movimientos sociales y las ciudadanías como parte de los currículos formales de las Escuelas, lo que de alguna manera da luces respecto de la formación de trabajadores y trabajadoras sociales con mayores conexiones con su entorno, capaces de adquirir y ejercer competencias actitudinales, cognitivas y procedimentales relacionadas con el respeto de los derechos de los pueblos.

Resulta contradictorio el cruce entre los perfiles de egreso y las mallas presentadas, puesto a pesar de contar con formación específica en derechos humanos, y asumiendo que estos son presentados de forma transversal en asignaturas como las prácticas, ética y otras, este sello profesional, este principio rector no es evidenciado en los perfiles de egreso. De esta forma, podríamos suponer que la ausencia de los derechos humanos, o si quiera el enfoque de derechos en los perfiles a pesar de considerarse en las mallas y planes de estudio, podría deberse a una acción deliberada en pos de atraer perfiles de ingreso altamente 
sensibles a las conjeturas del mercado. Lo anterior, aclararía la inconsistencia entre el compromiso desplegado en el perfil y su expresión en la malla, puesto que son estos elementos más sensibles, pertenecientes a lo que entendemos como proyecto ético político del trabajo social los que quedan ausentes en las declaraciones suscritas por las instituciones de educación superior.

La consideración de los derechos humanos en los perfiles de egreso y estructuras curriculares permite formar profesionales que ejerzan como sujetos de derecho, intencionado de esta forma las competencias y habilidades referidas a la toma de decisiones en escenarios complejos, propios del trabajo social, en especial cuando estas decisiones comprometen la dignidad y libertad de las personas.

El apostar por la formación de profesionales que tengan como características el ser sujeto de derechos los convertirá en personas capaces de

[...] equilibrar los derechos a la igualdad con los derechos a la diferencia y de ser un agente de cambio que combata la exclusión social, cultural y política, la segregación, el desarraigo, las injusticias e inequidades, las asimetrías sociales, las discriminaciones, los prejuicios y estereotipos, el racismo y la xenofobia, los choques culturales y sociales derivados de la falta de reconocimiento mutuo. (Magendzo, 2011, p. 113)

Traemos aquí el gráfico realizado por el mismo autor de la cita anterior respecto de la importancia de las competencias genéricas relacionadas con los derechos humanos para forjar a profesionales ciudadanos y ciudadanas sujetos de derecho.

De esta forma, la incorporación de competencias genéricas relacionadas con los derechos humanos permitirá y facilitará la formación de profesionales que sean agentes activos de la democracia, la capacidad de elección, el respeto a la dignidad y diversidad, y corresponsables del bien común. Lo anterior permite el cumplimiento de visiones que comprometen el reconocimiento de los egresados y egresadas por sus capacidades y compromiso ético, ética que al ser aplicada ve su mayor concreción en el respeto, promoción y defensa de los derechos humanos. 


\section{Ilustración 1. Competencias genéricas para la formación de un sujeto de derechos}

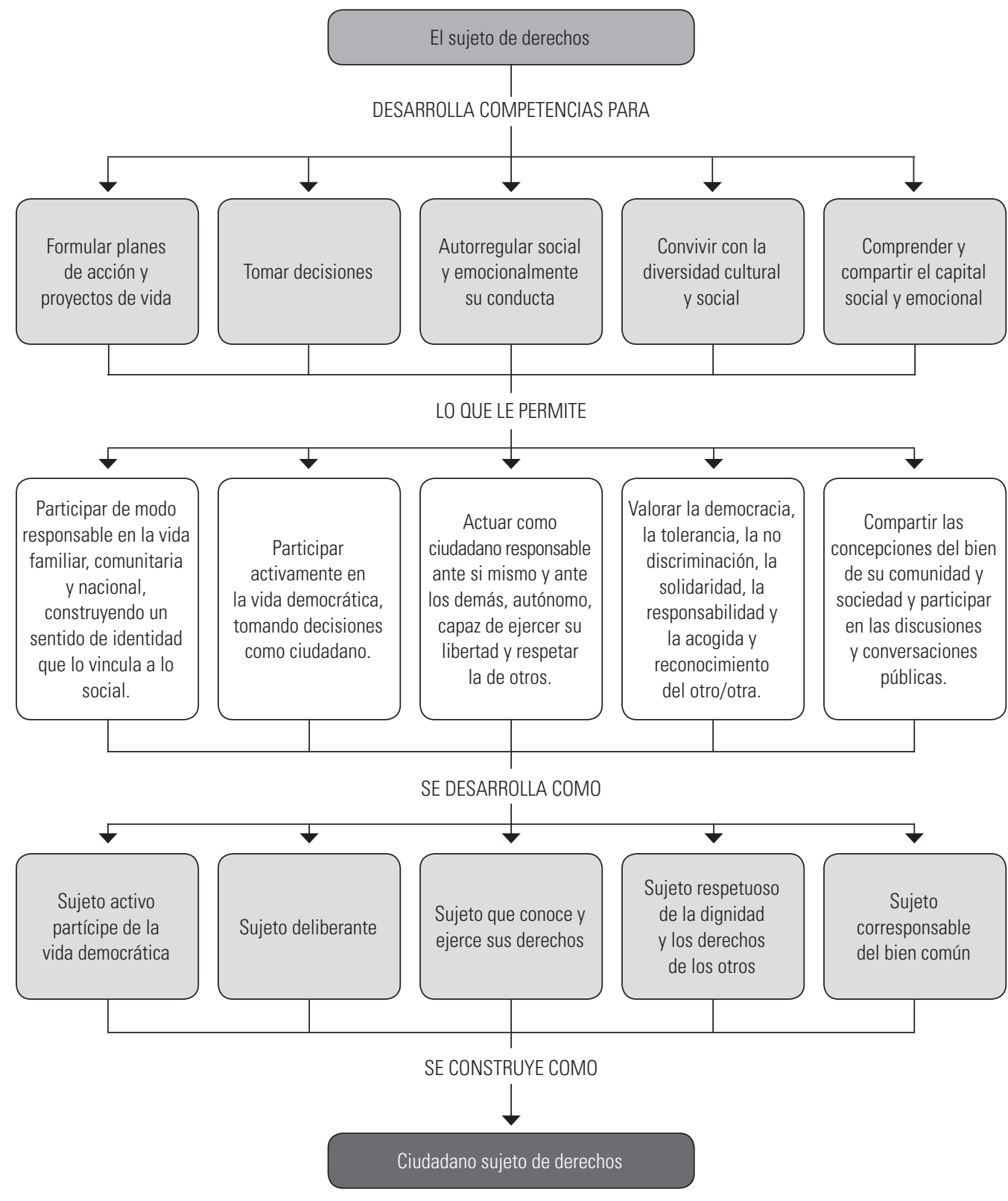

En: Magendzo, A., 2011, p. 118. 


\section{Conclusiones y reflexiones}

La educación en Derechos Humanos es un imperativo ético, no sólo para los y las profesionales de lo social, sino también una necesidad tendiente al fortalecimiento de las ciudadanías. Hoy en día, y según nuestra experiencia, los y las estudiantes observan con extrañeza la sola idea de hablar de Derechos Humanos. Extrañeza, puesto que hablamos de jóvenes que en su gran mayoría acceden a bienes y asocian la concepción de derechos a aquellos propios de los consumidores.

En este sentido, consideramos relevante la inclusión de asignaturas que expliciten la relación entre la profesión y los Derechos Humanos. Este tipo de asignaturas se configuran como un elemento basal en la formación de los y las trabajadoras sociales, pretendiendo estudiar en profundidad la noción de derechos humanos y los diversos problemas éticos y prácticos que ésta plantea, vinculándolos con el ejercicio profesional, relacionando la noción de Derechos Humanos con la intervención histórica y situada realizada en trabajo social.

Creemos que realizar un reconocimiento explícito respecto de la importancia de los derechos humanos como parte fundamental en un proyecto ético político en trabajo social, sentado en la democracia, y en lo que Montaño recoge como "progresista", permite formar profesionales con un alto compromiso ético, y protagonistas de sus propios procesos de subjetivación.

Los perfiles de egreso y las mallas de estudios constituyen declaraciones políticas de lo que la academia proyecta como definitorio y necesario en la formación de profesionales, encontrándose ante la tensión entre el mercado que condiciona, coarta y seduce; y las definiciones ético políticas esenciales para la formación en trabajo social.

Dado lo anterior, cabe preguntarse respecto de quién es la voz proyectada en los perfiles de egreso y mallas curriculares: ¿es la voz del medio?, ¿de los egresados y egresadas, del mercado laboral, de los académicos y académicas, de las organizaciones profesionales?

Creemos que responder a estos cuestionamientos, y reflexionar respecto de estas cuestiones permite avanzar hacia la construcción de un proyecto ético en el que los Derechos Humanos tengan un rol principal. 
Sin intentar apropiarse de una expertise en el tema, se presentan algunas recomendaciones a considerar en el tratamiento de los Derechos Humanos en las políticas públicas de educación superior en general y en las escuelas de trabajo social en particular.

Se hace necesario, dado el contexto actual chileno, el fortalecer la política pública de educación superior para que ésta establezca como elemento transversal en los currículos universitarios la educación en Derechos Humanos, con especial énfasis en la educación para la paz, la ciudadanía y la democracia, difundiendo a la vez, los instrumentos de Derechos Humanos suscritos por el país, con tal de contar con profesionales y técnicos que sean capaces de considerar el marco de análisis de los derechos humanos en sus actuaciones.

Así también, se recomienda considerar en los planes de estudios de las carreras relacionadas con lo social y lo jurídico, asignaturas que consideren los derechos de niños y niñas, la igualdad entre hombres y mujeres, la lucha contra la discriminación y la xenofobia, el derecho a la diversidad, el respeto a los pueblos originarios, y todos aquellos derechos consignados en la carta Internacional de Derechos Humanos.

En el mismo sentido se propone la incorporación del enfoque de derechos, en el diseño, ejecución y evaluación de políticas públicas y sociales ejecutadas por la plataforma estatal, considerando para esto el Pacto Internacional de Derechos Económicos, Sociales y Culturales.

En el caso de las escuelas de trabajo social, se recomienda incorporar cursos de Derechos Humanos, explicitando así su importancia y trascendencia para el cumplimiento del perfil profesional, y aportar a la construcción del proyecto ético político del trabajo social. Lo anterior requiere de estipular misiones y principios institucionales que den cuenta de la relación intrínseca entre derechos humanos y trabajo social, en otras palabras, se requiere declarar con fuerza y convicción la necesaria inclusión de los derechos humanos en los planes de estudios de trabajo social para así formar a profesionales que sean sujetos de derechos y contribuyan a que otros y otras también lo sean.

En el plano relacional, es necesario abogar por la instalación de relaciones, procesos y procedimientos con base en los derechos humanos al interior de cada una de las instituciones. 
En la formación de posgrado es preciso generar planes de formación que entreguen elementos profesionalizantes de profundización en Derechos Humanos, permitiendo fortalecer la investigación social en esa temática.

Finalmente, se requiere acciones que permitan formar a los trabajadores y trabajadoras sociales como sujetos de derechos y promotores de los mismos, incentivándoles a considerar los Derechos Humanos como el marco de actuación, el guión emancipatorio de las intervenciones, investigaciones y sistematizaciones realizadas en trabajo social.

Recebido em 8/4/2014 - Aprovado em 2/6/2014

\section{Referencias bibliográficas}

AZUA, Enrique. La educación en Derechos Humanos. En: SEMINARIO INTERNACIONAL UNIVERSIDAD Y DERECHOS HUMANOS, Actas..., Santiago de Chile, Instituto Nacional de Derechos Humanos, 2011. p. 31-37.

AIETS-FITS. Estándares globales para la educación y capacitación del trabajo social. Documento final en preparación para discusión, y posible adopción en las Asambleas Generales de AIETS y FITS, Adelaide, Australia en 2004, 2003. Disponible en: <http:// www.cpihts.com/pdf03/globalstandards\%202.pdf>.

CASTAÑEDA MENESES, P.; SALAMÉ, A. M. Construcción colectiva del perfil académico profesional en trabajo social. La experiencia de las Escuelas de Trabajo Social del Consorcio de Universidades Estatales. Revista Electrónica de Desarrollo de Competencias (REDEC), v. 1, n. 5, p. 42-71, 2011. [on-line] Disponible en: $<\mathrm{http}: / /$ redec. utalca.cl/index.php/redec/article/view/39/43>.

CORDERO, N.; PALACIOS, J.; FERNÁNDEZ, I. Trabajo social y derechos humanos: razones para una convergencia. Acciones e investigaciones sociales, Zaragoza, Universidad de Zaragoza, Facultad de Ciencias Sociales y el Trabajo, n. 1, p. 228, 2006.

CORTINA, Adela. El estatuto de la ética aplicada. Hermenéutica crítica de las actividades humanas. Isegoría, Madrid, Instituto de Filosofía/Consejo Superior de Investigaciones Científicas, n. 13, p. 119-127, 1996. 
CORTINA, Adela. La dimensión pública de las éticas aplicadas. Revista Iberoamericana de Educación, n. 29, p. 45-64, 2002.

DECLARACIÓN DE MÉXICO SOBRE EDUCACIÓN EN DERECHOS HUMANOS EN AMÉRICA LATINA Y EL CARIBE. Ciudad de México, 28 de noviembre $-1^{\circ}$ de diciembre de 2001. Disponible en: <http://iidh-webserver.iidh.ed.cr/multic/UserFiles/ Biblioteca/IIDH/2_2010/AspecTeoMetodologico/Material_Educativo/Declaracion-Mexico.htm>.

HAWES, Gustavo. El perfil de egreso. Departamento de Educación en Ciencias de la Salud. Facultad de Medicina, Universidad de Chile (Documento de trabajo), 2012. Disponible en: $<$ http://www.gustavohawes.com/Educacion\%20Superior/2010Perfil\%20 de\%20egreso.pdf $>$.

INSTITUTO NACIONAL DE DERECHOS HUMANOS. Informe Anual Situación de los Derechos Humanos en Chile. Santiago de Chile: Instituto Nacional de Derechos Humanos, 2012.

INTERNATIONAL FEDERATION OF SOCIAL WORKERS/INTERNATIONAL ASSOCIATION OF SCHOOL OF SOCIAL WORK. Ethics in Social Work: statement of principles, 2004. Disponible en: <www.ifsw.org $>$.

INTERNATIONAL FEDERATION OF SOCIAL WORKERS, EUROPE REGIONA. Standards in Social Work Practice Meeting Human Rights, 2010. Disponible en: $<$ http:// cdn.ifsw.org/assets/ifsw_45904-8.pdf \$.

KRIPPENDORFF, Klaus. Metodología del análisis de contenido: teoría y práctica. Barcelona: Paidós Ibérica, 1990.

MAGENDZO, Abraham. Educación en derechos humanos, un desafío impostergable. En: COMISIÓN NACIONAL CHILENA DE COOPERACIÓN - UNESCO. Políticas públicas para la infancia. Santiago de Chile: Unesco, 2011. p. 101-119.

MONTAÑO, Carlos. Hacia la construcción del proyecto ético-político profesional crítico. In: . Búsquedas del trabajo social latinoamericano: urgencias, propuestas y posibilidades. Buenos Aires: Espacio Editorial, 2005. p. 39-48.

MUJICA, Rosa María. La metodología de la educación en derechos humanos. Costa Rica: Instituto Interamericano de Derechos Humanos, 2002. Disponible en: $<\mathrm{http} / /$ www.egdhdih.mil.do/lecturas $\% 20 \mathrm{y} \% 20$ textos $\% 20 \mathrm{de} \% 20 \mathrm{ddhh}$-dih/derechos $\% 20$ humanos/derechos $\% 20$ humanos $\% 20 \mathrm{y} \% 20$ educacion/2.pdf $>$. 
NACIONES UNIDAS. Asamblea General. "Declaración y Programa de Acción de Viena”. A/CONF.157/23, 12 de Julio de 1993.

. Derechos humanos y trabajo social. Manual para escuelas de Servicio Social y trabajadores sociales profesionales. Ginebra: FITS-Consejo Derechos Humanos Naciones Unidas, 1995. (Serie de capacitación profesional, n. 1.) Disponible en: $<$ http:// cdn.ifsw.org/assets/ifsw_104630-7.pdf>.

NASH, Claudio. Diagnóstico sobre la incorporación de los derechos humanos en las carreras de Derecho en Chile. En: SEMINARIO INTERNACIONAL UNIVERSIDAD Y DERECHOS HUMANOS, Actas..., Santiago de Chile, Instituto Nacional de Derechos Humanos, p. 61-72, 2011.

LÓPEZ NOGUERO, Fernando. El análisis de contenido como método de investigación. XXI: Revista de Educación, Universidad de Huelva, n. 4, p. 167-199, 2011. Disponible en: $<$ http://www.uhu.es/publicaciones/ojs/index.php/xxi/article/view/610/932>.

OLAYA, Eucaris. Perspectiva ético-política en la investigación e intervención de Trabajo Social. Revista de Servicio Social, 2009. Disponible en: $<$ http://www.uel.br/revistas/ssrevista/pdf/2008/25\%20PERSPECTIVA\%20\%C9TICO-POLITICA\%20OCTUBRE\%2008.pdf>.

PORTA, L.; SILVA, M. La investigación cualitativa: el análisis de contenido en la investigación educativa. Red Nacional Argentina de Documentación e Información Educativa, 2003. Disponible en: <http:/www.uccor.edu.ar/paginas/REDUC/porta.pdf $>$. Acceso en: 1 feb. 2010.

RAMÍREZ, Gloria. Los caminos de la educación superior en derechos humanos en México. Hacia una política pública. En: MAGENDZO, Abraham (Ed.). De miradas y mensajes en la educación en Derechos Humanos. Santiago de Chile: LOM Ediciones, Cátedra Unesco de Educación en Derechos Humanos, Universidad Academia de Humanismo Cristiano, 2003.

RAIGADA, José Luis Piñel. Epistemología, metodología y técnicas del análisis de contenido. Estudios de sociolingüística: Línguas, Sociedades e Culturas, Pontevedra, Universidad de Vigo, v. 3, n. 1, p. 1-42, 2002.

SANTOS, Boaventura de Sousa. Hacia una concepción multicultural de los derechos humanos. El Otro Derecho, ILSA, Bogotá, Colombia, n. 28, p. 59-83, jul. 2002.

. El milenio huérfano. In: . Ensayos para una nueva cultura política. Madrid: Trotta, 2005. 
TÓJAR, Juan Carlos. Investigación cualitativa: comprender y actuar. Madrid: La Muralla, 2006.

VERLOO, Mieke. Mainstreaming Gender Equality in Europe. A frame analysis approach, en: The Greek Review of Social Research, B'117, p. 11-13, 2005 [on-line]. Disponible en: <http://www.grsr.gr/pdf/117_11-34.pdf>.

VERLOO, M.; LOMBARDO, E. Contested gender equality and policy variety in Europe: introducing a critical frame analysis approach [on-line], 2007. Disponible en: $<$ www.ru.nl/publish/pages/.../verloo_multiple_meanings_intro_2007.pdf > 